\title{
The unreachable doorbells of South Texas: community engagement in colonias on the US-Mexico border for mosquito control
}

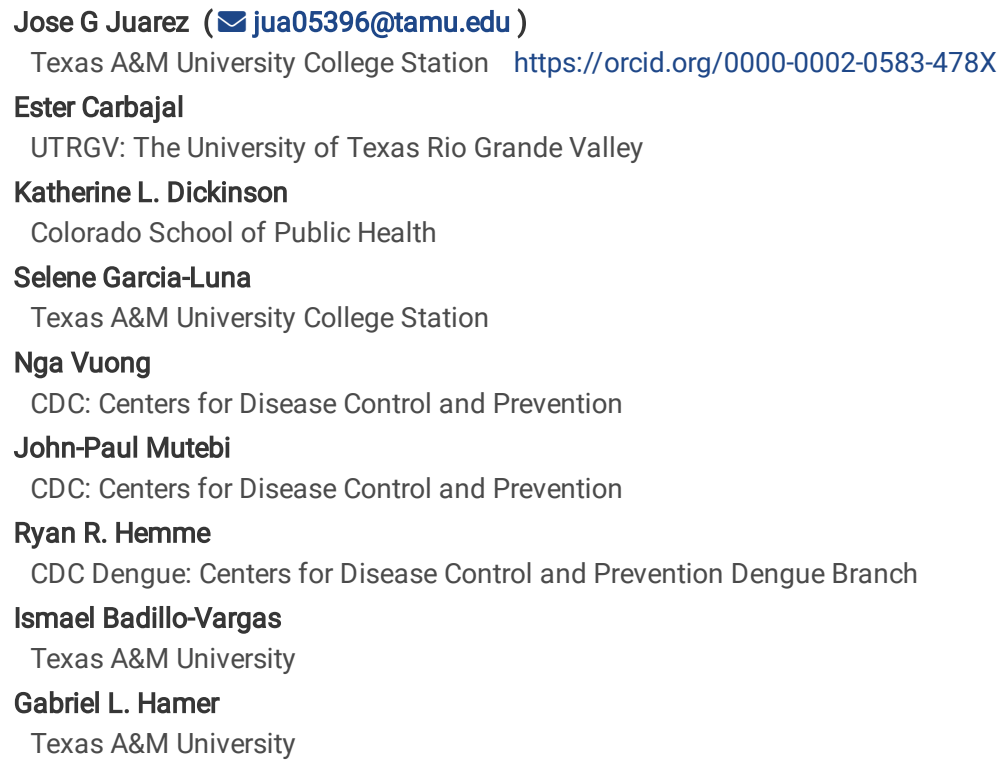




\section{Abstract}

Mosquitoes and the diseases they transmit continue to place millions of people at risk of infection around the world. Novel methods of vector control are being developed to provide public health officials with the necessary tools to prevent disease transmission and reduce local mosquito populations. However, these methods will require public acceptance for a sustainable approach and evaluations at local settings. We currently present our efforts in community engagement carried out in colonias of the Lower Rio Grande Valley in south Texas for mosquito surveillance, control, and ecological projects. Along the USMexico border the term colonia refers to impoverished communities that are usually inhabited by families of Hispanic heritage. The different engagements were carried out from September 2016 to February 2019; during this time, we had three distinct phases for community engagement. In Phase 1 we show the initial approach to the colonias, Phase 2 the first recruitment procedure, and Phase 3 a modified recruitment procedure based on community members' input. Our results show that incorporating community members in the development of communication materials and following their suggestions for engagement allowed us to generate culturally sensitive recruitment materials and to better understand the social relationships and power dynamics within these communities. We were able to effectively reach a larger portion of the community and decrease the dropout rate of participants. Community engagement should be viewed as a key component of any local vector control program as well as for any scientific research project related to vector control. Even in the face budgetary constraints, small efforts in community engagement go a long way.

\section{Background}

Mosquitoes are vectors of human parasitic and viral diseases that affect millions of people per year around the world [1]. They cause the highest burden of disease transmission to humans by an arthropod vector [2] and are a major public health threat [3]. In the case of container Aedes mosquitoes and associated Aedes-borne viruses like dengue and Zika, traditional vector control programs have fallen short [4,5], partially because of population growth in urban areas, connectivity between communities [6], climate change [7], the ability of Ae. aegypti to adapt to urban environments [8] and insecticide resistance [9]. Surveillance of Aedes mosquitoes is a key component of any vector control program, but success varies depending on the type of surveillance and control tools used [10].

From the early 1950 s to late 1980 s, centralized control activities were very successful at reducing Aedes aegypti population in the Americas [11] and malaria transmission in Africa [12], as well as almost eliminating onchocerciasis transmission in West Africa [13]. However, the impact of these programs waned over time due to insecticide resistance, difficulty accessing houses (i.e., unwillingness to allow unknown technicians into homes and the smell of insecticides [14]), and lack of sustained investments [15]. Since the late 1990s, vector control programs in the Americas have become more decentralized, focusing on smaller areas, and using a bottom-up approach. More recently, programs have begun to emphasize engagement with community members and stakeholders as part of control efforts to improve long term sustainability of a project and help during control activities [16-18].

In the contiguous United States of America (USA), there are very few regions that have both presence of Ae. aegypti and local transmission of Aedes-borne viruses such as dengue or Zika. One of these regions is the Lower Rio Grande Valley (LRGV) located in south Texas [19]. Within the LRGV, mosquito control programs follow a decentralized regimen where the local cities or counties are responsible for surveillance and control [20]. In this region, vector control activities are minimally funded with an estimated $\$ 0.05$ per person contributing to the vector control budget in Hidalgo County [21].

Within the LRGV region, there are over 1,800 unincorporated communities known as colonias which are usually inhabited by families of Hispanic heritage who often live in low-income housing and lack essential city services such as waste management, paved roads and potable water [22-24]. These systematic disparities create conditions that are favorable for Ae. aegypti proliferation and is unfavorable for the health of community members due to deficits in multiple social determinants of health [25]. Colonias also have issues with social cohesiveness due in part to vacant lots [26] which contributes to them being a hardto-reach minoritized group [27]. These factors present barriers to engaging effectively with communities to implement vector control interventions [18, 28] in the precise environments where these interventions are most needed. Here, we present our team's experience with community engagement approaches in the context of vector control in colonias in the LRGV. We present evidence that working with community leaders, following community members' suggestions, and making subtle changes to engagement techniques improved community willingness to participation in our projects and reduced dropout rate.

The inclusion of behavioral and social sciences into public health interventions can't be overlooked, they provide critical guidance when developing a project that depends on communities and public acceptance. Lessons here can inform broader efforts to work with communities to implement more effective vector control approaches, showing the importance of getting early community buy-in and support ahead of time.

\section{Main Text}

\section{Study area}

Our research took place in Hidalgo County, located in the LRGV region along the US-Mexico border of South Texas, USA (Figure 1). Within this county, there are an estimated 0.8 million people of which $90 \%$ are of Hispanic origin, $28 \%$ live below the poverty line and $19 \%$ are foreign-born individuals [29]. This region has three major points of entry into the US (Hidalgo, Progresso and Brownsville), and during the duration of the studies, there were over 23 million and 28 million recorded crossings for 2017 and 2018, respectively [30].

\section{The projects}

Within the study area we conducted four projects that required the participation of community members. These projects were conducted between September 2016 and December 2018. We had two intervention projects and two ecological studies focused on Ae. aegypti. The intervention projects evaluated two novel traps, the Autocidal Gravid Ovitrap (AGO) (this project has been previously detailed in Juarez et al, [28], see Supplementary Information: AGO project, for a brief 
description) and the Autodissemination Station (ADS), for mosquito suppression and field performance under local conditions (see Supplementary Information: Vector control traps [31]). The ecological projects involved the isotopic enrichment of container habitats to evaluate mosquito dispersal [32] and cryptic habitats. Each of these projects relied heavily on community participation, since we required permission from homeowners to enter their properties to set up traps, for either control or surveillance, and search for larval habitats. Figure 2 shows the timeline and activities carried out for each project with three key activities: A) Phase 1, B) Phase 2, and C) Phase 3. We further expand on each of these key activities in Table 2.

\section{Team and expertise}

We were composed of a multidisciplinary team of local and international personnel, including student workers from a local University (University of Texas Rio Grande Valley). The expertise of our core team members varied and included local community health workers (known as Promotoras); local community members; members with knowledge in community engagement with expertise in Neglected Tropical Diseases; and subject matter experts in the field of mosquitoes and vector-borne diseases. Most of our core team were native Spanish speakers and fluent in English. The lived experience from our local team members provided a unique perspective into the mindset of participants living in the LRGV and the colonias. We leveraged this knowledge to help us understand how community members perceived outsiders and local authorities. Combining the multiple perspectives of our team we were able to develop engagement activities, educational material and surveys that were culturally appropriate and tailored for the colonias. This allowed us to address issues of colloquialisms and the inclusion of community members during development of these tools.

\section{Community selection}

Community selection has been previously detailed in other studies [32-34]. Briefly, we used the 2010 census blocks to identify colonias based on a mean household income of $\$ 15,000-\$ 29,999$ that were within a $30 \mathrm{~km}$ radius from our field station (Texas A\&M AgriLife Research and Extension Center in Weslaco, Texas) (Figure 1). The identified colonias were selected based on size (e.g., range of 20 to 150 households), level of isolation from other communities or urban landscapes, and perception of safety for field personnel based on comments from local state officials. Seventeen colonias from Hidalgo County were initially visited for evaluation and six were selected based on security for field personnel, willingness of community members to participate, and access to the community. These colonias were distributed within the cities of Donna $(n=2)$, Progresso $(n=1)$ and Mercedes ( $n=3)$; four communities (e.g., Balli, Mesquite, Chapa and Cameron) were involved in the Autocidal Gravid Ovitrap (AGO) project (Figure 1C) and two (Indian Hills West and La Piñata) were involved in the ecological and Autodissemination Station (ADS) projects (Figure 1D).

\section{Community members}

Our different projects categorized community members into two types: engages and participants. Engages had weekly visits to check surveillance traps, participated in interviews and surveys, and participated in the development of fliers. Personal information was only collected from the individuals involved in the AGO project and used in the analysis of mosquito risk factors [31]. Participants were all the remaining houses within each colonia that received the intervention and had visits on a monthly or bi-monthly schedule. We did not collect any type of personal information from these individuals (Table 1 ). If an engage dropped out, we tried to recruit its neighbor to the right until a new one was recruited; if an engage dropped out they were still invited to join the project as participants. To identify all possible houses within each community, we georeferenced all structures using Google satellite imagery (Google, Maxar Technologies) in QGIS 2.8 (https://qgis.org/en/site/) and confirmed them with field visits.

Table 1. Colonias of the LRGV with the projects carried out (Autocidal Gravid Ovitrap=AGO; Autodissemination Station=ADS), year of complete community recruitment, total active houses during the recruitment, engages and participants.

\begin{tabular}{llllll} 
Community & Project & Year & Total active houses & Engages & Participants \\
\hline Balli & AGO & 2017 & 40 & 7 & 18 \\
\hline Cameron & AGO & 2017 & 78 & 6 & 35 \\
\hline Chapa & AGO & 2018 & 27 & 5 & 19 \\
\hline Mesquite & AGO & 2018 & 37 & 5 & 26 \\
\hline La Piñata & Dispersal & 2017 & 151 & 50 & - \\
\hline & ADS & 2018 & 146 & 15 & 84 \\
\hline Indian Hills West & Cryptic cont. & 2017 & 79 & 32 & - \\
\hline & ADS & 2018 & 82 & 10 & 50
\end{tabular}

\section{Phase 1}

Autocidal Gravid Ovitrap ( $(\underline{A G O})$ intervention: This project had a rigorous site-selection procedure in the initial approach phase from September 2016 through June 2017. In this initial phase, several engages objected to the odor produced by the AGO traps both indoors and outdoors, leading to eight dropouts. In total we had a 44\% (16/36) dropout rate during phase one [34]. With the input from engages, we adjusted the dose of hay in the trap so that the odor did not disturb residents of the home while still attracting female mosquitoes (Table 2). Engages were randomly selected within each colonia with written consent provided by one of the adult household participants [31, 34]. Due to the long duration of the AGO project (September 2016 to December 2018 ), the recruitment was ongoing since engages could drop out of the study at any point. 
Ecological projects (dispersion and cryptic containers): The colonias of La Piñata and Indian Hills West did not have a rigorous site selection procedure and focused on dispersion and cryptic containers of mosquitoes, respectively. We initially approached the communities in July of 2017 (see Supplementary Information: ecological projects, for a brief description). As such, we increased engagement activities related to information sharing (see Supplementary Figure S1) and consultation with community members. In both projects, we intended to recruit as many households as possible within each colonia and randomly selected the engages. We noticed that these communities had some form of social integration between community members that we did not observe in the AGO colonias. We observed that within these colonias, there were several group leaders, either by family association or that had a common interest (i.e., mothers from school children, social friends, etc.). The members within these groups would have regular communication with each other by either WhatsApp groups, a mobile communication application, or visits to each other's house. This knowledge allowed us to coordinate community meetings with some of the sectors, present the projects, discuss its limitations, and alleviate doubts from community members. During this process, we identified two key aspects of these colonias. Firstly, the communities had different groups/sectors, some of which did not interact with each other. Secondly, some of these groups/sectors had a leader whose approval was necessary to effectively recruit households within each sector. As an example, one community member asked our team, "Did the house in the corner agree? If so, you can place the traps" which suggested that approval from the leader was necessary. However, we still relied heavily on house-to-house recruitment since these groups/sectors were not applicable to most of the Colonia.

\section{Phase 2}

Autocidal Gravid Ovitrap (AGO) intervention: In the second phase of recruitment, we visited participants households within each community at least three times (e.g., morning-afternoon-weekend), giving them an equal opportunity to join the project (Table 2). We approached community members, conducted trap demonstrations on-site and reviewed the different procedures carefully in either English or Spanish, as requested. During the recruitment procedure, we used an informative flyer (Figure 3A) that served two purposes: 1) provided an overview of the project with our contact information to community members and 2) served as a signal to our recruitment team if a house was unoccupied. If no-one was found in the house during the first visit, we left the flyer hanging on a visible area of the door (e.g., above the doorknob) or gate (e.g., in the lock); if this signal remained in the same place after the third visit, we considered the house empty. On several occasions, the flyer was picked up and voices were heard inside of the household, but no one responded directly to our knocks.

In 2017 after the third house-to-house visit, we were able to recruit only between $48 \%$ and $55 \%$ of the available households. Informal discussions with some community members that were recruited as participants for 2017 showed that some of them did not open the door during the first visit because they thought we were either 1) selling something, 2) debt collectors, or 3) members of a religious organization. All team members and visiting scholars always wore university associated clothing and avoided green colored attire to decrease the perception that the team was associated with the Border Patrol.

Long-term enrollment of engages ( $\underline{A G O})$ : Our goal was to maximize retention of engages and keep them involved throughout the study. We visited those households every week during the study period except for the last two weeks of December and the first week of January, due to the holidays. Weekly visits lasted between 15 to 45 minutes depending on conversations we had with engages. Topics ranged from the perception of their community, our study, local vector control activities, and personal issues they encountered during the week. As part of our strategy for retaining households, we provided information about seasonal mosquito abundance in their home, community, and region. This information was given anytime the homeowner requested it, as well as to all households at the start of 2018 and after the project ended in 2019.

To better understand the perception of engages regarding mosquitoes, including their diseases and control measures, and our project, we carried out a Knowledge, Attitude and Practices (KAP) survey in November 2017 and 2018 [31]. The results obtained from the 2017 KAP showed that community members considered the use of television and flyers as the best methods for communication in the colonias. The KAP also allowed us to generate an initial draft of an informative flyer to work with community members and show the results of our project (see Supporting Figure S2A - B). The final version showcased information that community members perceived as critical, such as increasing the size of the images used for the seasonality, emphasizing the Centers for Disease Control and Prevention (CDC) webpage, and showing the actual size of the mosquitoes we were studying. They also suggested decreasing the amount of text and increasing the size of the greeting message. This flyer was distributed in February 2018 to all community members in the different projects regardless of involvement in the studies. Overall, community members appeared more receptive to this flyer, even those that had not participated in the project were interested in having us explain the flyer and our activities. We even had some engages that requested more than one flyer so they could show it to other people. Finally, we provided engages with a \$5 gift card from a local supermarket on four occasions, two times in both 2017 and 2018 . These were provided in August and December as a token of appreciation to homeowners for their consistent support. In phase two we had a 4.45\% (2/44) dropout rate [28], which was a $90 \%$ decrease in dropout compared to Phase 1.

Ecological projects (dispersion and cryptic containers): We recruited engages as described for the AGO project, with up to three house-to-house visits. The results of the recruitment process in the communities of La Piñata and Indian Hills West were like those observed for the AGO project. We recruited $42 \%$ and $49 \%$ of community members in La Piñata and Indian Hills West, respectively. The first visit had an average of $25 \%$ community recruitment, with an average of $13 \%$ and $9 \%$ for the second and third visit respectively. The recruitment results were like those obtained in the AGO communities. Overall, these showed us that the recruitment method for Phase 2 was not sufficient to effectively reach most community members.

\section{Phase 3}

Autocidal Gravid Ovitrap ( $(\underline{A G O})$ intervention: We slightly modified the recruitment process by conducting the third visit after 5:00 pm and developing a shorter recruitment flyer (Figure $3 \mathrm{~B}$ ) based on feedback from participants to improve aesthetics, clear objectives of the project, and clarity of words. The first recommendation from community members was to make it clear that the trap was free and to clarify the procedures involved, such as a reset visit every one or two months by the study team. Another key comment was to clarify the safety of the trap. In 2018 , we were able to recruit between $81 \%$ and $86 \%$ of households in the selected colonias into the AGO study. Comparing recruitment for Phase 2 and Phase 3, we had similar results for the first visit (average of $37 \%$ and $32 \%$ respectively), but saw a large increase in recruitment at the second visit with an average of $47 \%$ in 2018 compared to an average of $13 \%$ in 
2017. (Figure 4). Interestingly, in 2018, we had to re-enroll $2 \%$ of households during the October reset since we encountered different participants at that time. In some cases, participants wondered what the traps were for and in others, they had already been told about our project. We believe some of these households host transient populations that might only spend a short period of time in the colonias.

Autodissemination station (ADS) intervention: For this project, the recruitment process started in early July 2018 . We began by holding community meetings to ensure the purpose of the study was well understood and to confirm that community leaders had not changed (see Supplementary Information: ADS project, for a brief description). We obtained authorization from community members to conduct the study and agreed to follow community recommendations regarding engagement activities on how to approach the different groups found within each community. In contrast to the AGO study, both the engages and participants were recruited simultaneously. As in the AGO study, we got active feedback from community members regarding the recruitment flyer (Figure 5). The community meetings allowed us to determine the need for extra engagement activities in the community of La Piñata such as a science tent at the entrance of the community, which was deployed the Saturday before the house-to-house visits started. This tent had information regarding our study, the ADS trap, live mosquito larvae and activities for children (Table 2).

The recruitment procedures for both groups, engages and participants, were the same and each household was visited at least three times. We were able to recruit $51 \%$ and $60 \%$ of community members in La Piñata and Indian Hills West, respectively. When comparing the recruitment of 2017 to that of 2018 we observed an increased average of $30 \%$ on the first visit of 2018 (Figure 6). As with the 2018 AGO project we had to re-enroll $5 \%$ of households when we arrived to service the traps and noticed that there were different participants living in the house. Re-enrollment usually happened after two months of the traps being deployed.

Table 2. Overview of approaches for Phases 1, and recruitment for Phases 2 and 3. 


\begin{tabular}{ll} 
Phases & Highlights \\
\hline 1 & $\begin{array}{l}\text { Colonias were selected based on security, willingness to participate from community members and access to the } \\
\text { community. During this initial phase we tried to identify community leaders that might help us during engagement and } \\
\text { recruitment activities. }\end{array}$ \\
& $\begin{array}{l}\text { We were unable to identify community leaders in the colonias where the Autocidal Gravid Ovitrap intervention took } \\
\text { place. Instead, we encountered pockets of familial households living in proximity. We observed that community } \\
\text { members in these colonias rarely interact with each other, and many times they would not know their next-door } \\
\text { neighbors. Interestingly, some community members emphasize their unwillingness to participate in community } \\
\text { meetings or talk to their neighbors and stated that they would rather not be involved in the study if that was the case. }\end{array}$
\end{tabular}

We did identify community leaders in the colonias were the Ecological and Autodissemination studies took place. We observed several leaders by Colonia with some having a well-defined sector. These were usually formed by community members that had some type of association between each other (i.e., family related, mothers with children of similar age, social friends, etc.). Similarly, we observed a characteristic unwillingness of community members to participate if different sectors had to interact. In some cases, we were even asked why certain sectors of the community had to participate in the study.

Phase We approached all the households at least three times (morning-afternoon-weekend). If the homeowner was not found $2 \quad$ in the first visit, we left an informative flyer in the door or gate (Figure 3 and 5).

We carried out sectorized community meetings ( $<5$ people) in the colonia of the dispersal project. In some cases, a homeowner would approve the project, but required confirmation that the sector leader approved of the activities.

We conducted a Knowledge, Attitude, and Practices (KAP) survey in the AGO colonias. Which provided us with a better understanding of the perceptions of community members regarding mosquitoes and how to improve engagement activities based on community knowledge gaps and specific interests. We also carried out informal conversations with participants regarding their hesitance to answer the door during the first approach visit.
Key lessons

Not forcing community meetings or interaction between community members.

Adjusting the traps used to fit the requirements of participants.

Following the security recommendations of community members regarding activity time of day.
The recruitment flyer served two purposes: provide information of the project and a signal for household occupancy.

Colonias have very different social dynamics, even for those that are geographically close.

Requesting personal information can negatively affect the participation rate of community members.
Phase We focused on collaborating with different community members, prior to the house-to-house recruitment, to develop new recruitment flyers for both the AGO and ADS projects. Flyers were developed following the guidance and advice of community members regarding images, wording, and their interests. We emphasized project had no cost and clarified the procedures to be completed in their property.

In the colonia of La Piñata, we hosted a science tent in the community entrance, based on conversations we had during the sectorized community meetings. The meetings allowed us to identify the need for additional engagement activities in this community.

The house-to-house recruitment was modified to include the afternoon visit after 5:00 pm. All other recruitment procedures were as in 2017.
The use of a short recruitment flyer improved

participation rate in the colonias.

Inclusion of community members during the development of engagement material.

The visit after 5:00 pm didn't increase our participation rate.

\section{Strengths And Limitations}

The engagement of stakeholders and community members in public health interventions is key to improving the health and well-being of disadvantaged and marginalized groups [35]. For novel methods of vector control, early involvement of key participants may allow us to assess acceptability for new approaches 
and to detect problems that may lead to public rejection of certain technologies [36, 37]. More importantly, engaging with affected populations allows us to gain in-depth knowledge of the ecological, biological, political, and social complexities in which the novel vector control approach would be implemented. However, community engagement does not have a one size fits all framework that all projects could follow [38], even in situations like ours where communities are geographically close. Community engagement activities need to be culturally appropriate and sensitive to effectively reach community members from underserved populations [39] which might be affected by different disparities and barriers (e.g., budgetary constraints, social cohesiveness). From the different projects we conducted related to mosquito ecology and control, we show that even with a limited budget for community engagement, the inclusion of such activities allowed us to improve community participation, retention and engage transient community members in the colonias of the LRGV along the US-Mexico border in Texas.

Undertaking community engagement activities can feel overwhelming especially for local vector control programs that may lack trained personnel in the social and behavioral sciences field. Understanding what community engagement is might be confusing since there is a variety of definitions and models available for program implementation [36, 38, 39]. In the case of the colonias, external investigators might think that these community members would share the same perspectives or belief systems since most are of Hispanic heritage. However, the reality is that community members migrated from a range of different countries, and have different immigration statuses, ethnicities, languages, or dialects (e.g., North Mexican Spanish, Guatemalan Spanish, TexMex, etc.), and cultural nuances. This shows a clear need to understand the needs of our communities at a local level while taking into consideration that mistrust from marginalized communities is common [40] and was something that we were able to observe throughout our studies within the colonias.

In our study, we continuously worked with community members to develop culturally sensitive recruitment materials and to better understand the social relationships and power dynamics. Some limitations of our projects were that we were unable to fully involve community members in the design and type of intervention to be used. We had budgetary constraints for the inclusion of a larger sample of community members along the LRGV, as well as for the materials we could develop and use. Finally, our work has socio-geographic barriers that might not be seen elsewhere so we cannot generalize our results to all colonias along the US-Mexico border.

We observed that the inclusion of community members for the outreach material and the type of communication to use (flyers) proved very useful when evaluating the efficiency of the AGO intervention, which showed that this trap is dependent on density in an area that ultimately depended on community participation [28]. The inclusion of community engagement activities in our multiple projects increased stakeholder engagement and acceptability and allowed us to conduct robust science in communities that might be considered as hard to reach. This allowed us to also elucidate novel ecological features of mosquitos such as a longer range of dispersal for the region [32] and risk factors associated with female mosquito abundance [31]. These studies wouldn't have been possible to conduct without the support and willingness of community members to allow us to enter their homes either on a weekly, monthly, or bimonthly basis. Trust from participants was crucial since on many occasions we were authorized to go into their properties without them being present, providing us with consistency on our surveillance data.

\section{Conclusion}

The results drawn from these studies show that community engagement activities should be a key component of any vector borne disease research project, effective local vector control program, or other public health intervention within the LRGV and elsewhere. Among vector control programs with budgetary constraints, our studies show that small efforts in community engagement can have a positive impact on their mitigation efforts.

\section{Abbreviations}

\section{AGO}

Autocidal Gravid Ovitrap

ADS

Autodissemination Station

LRGV

Lower Rio Grande Valley

KAP

Knowledge Attitude and Practice

\section{Declarations}

\section{Ethic approval and consent to participate}

This project received approval from the Institutional Review Board of Texas A\&M University (IRB2016-0494D). We obtained individual written consent from each household owner for the weekly indoor and outdoor entomological surveillance.

\section{Availability of data and materials}

All data generated or analyzed during this study are included in this published article [and its supplementary information files].

\section{Competing interests}

The authors declare that they have no competing interests 


\section{Funding}

This work was supported by National Institutions of Health R21Al128953 and Cooperative Agreement Number U01CK000512, funded by the Centers for Disease Control and Prevention. Its contents are solely the responsibility of the authors and do not necessarily represent the official views of the Centers for Disease Control and Prevention or the Department of Health and Human Services.

\section{Author's contributions}

Conceptualization, J.G.J., K.D., and G.L.H.; methodology, J.G.J., E.C., S.G.L., and G.L.H.; validation, J.G.J., E.C., and S.G.L.; investigation, J.G.J., E.C., S.G.L., K.D., I.B.V., and G.L.H.; resources, I.B.V., and G.L.H.; data curation, J.G.J., E.C., S.G.L., K.D., N.V., J.P.M., R.R.H., I.B.V., and G.L.H.; writing-original draft preparation, J.G.J., E.C., and G.L.H.; writing-review and editing, J.G.J., E.C., S.G.L., K.D., N.V., J.P.M., R.R.H., I.B.V., and G.L.H.; visualization, J.G.J.; supervision, J.G.J., and G.L.H.; project administration, J.G.J. and G.L.H.; funding acquisition, G.L.H. All authors have read and agreed to the published version of the manuscript.

\section{Acknowledgements}

We are grateful to the residents of our study locations in the Lower Rio Grande Valley, that collaborated with us to conduct these projects in their neighborhoods. We thank Edwin Valdez, Courtney Avila, Cynthia Flores, Juliet Vallejo, Damion Blanchard and Daniel Zamarripa for their assistance in the field. We would like to thank the county of Hidalgo and the cities of McAllen and Weslaco, for their guidance provided during community selection.

\section{References}

1. World Health Organization. Global vector control response 2017 - 2030 A strategic approach to tackle vector-borne diseases. 2017;:00-3. http://apps.who.int/iris/bitstream/10665/259002/1/WHO-HTM-GVCR-2017.01-eng.pdf?ua=1. Accessed 23 Jan 2018.

2. Eder M, Cortes F, Teixeira de Siqueira Filha N, Araújo de França GV, Degroote S, Braga C, et al. Scoping review on vector-borne diseases in urban areas: Transmission dynamics, vectorial capacity and co-infection. Infect Dis Poverty. 2018;7. doi:10.1186/s40249-018-0475-7.

3. Franklinos LHV, Jones KE, Redding DW, Abubakar I. The effect of global change on mosquito-borne disease. Lancet Infect Dis. 2019;19:e302-12. doi:10.1016/S1473-3099(19)30161-6.

4. Reiner RC, Achee N, Barrera R, Burkot TR, Chadee DD, Devine GJ, et al. Quantifying the Epidemiological Impact of Vector Control on Dengue. PLoS Negl Trop Dis. 2016;10:e0004588. doi:10.1371/journal.pntd.0004588.

5. Tian H, Sun Z, Faria NR, Yang J, Cazelles B, Huang S, et al. Increasing airline travel may facilitate co-circulation of multiple dengue virus serotypes in Asia. PLoS Negl Trop Dis. 2017;11:e0005694. doi:10.1371/journal.pntd.0005694.

6. Campbell LP, Luther C, Moo-Llanes D, Ramsey JM, Danis-Lozano R, Peterson AT. Climate change influences on global distributions of dengue and chikungunya virus vectors. Philos Trans R Soc B Biol Sci. 2015;370:1-9. doi:10.1098/rstb.2014.0135.

7. Ferguson NM. Challenges and opportunities in controlling mosquito-borne infections. Nature. 2018;559:490-7. doi:10.1038/s41586-018-0318-5.

8. Deming R, Manrique-Saide P, Medina Barreiro A, Cardeña EUK, Che-Mendoza A, Jones B, et al. Spatial variation of insecticide resistance in the dengue vector Aedes aegypti presents unique vector control challenges. Parasit Vectors. 2016;9:67. doi:10.1186/s13071-016-1346-3.

9. Bowman LR, Runge-Ranzinger S, McCall PJ. Assessing the Relationship between Vector Indices and Dengue Transmission: A Systematic Review of the Evidence. PLoS Negl Trop Dis. 2014;8:e2848. doi:10.1371/journal.pntd.0002848.

10. CDC. The Aedes aegypti eradication program. 1966.

11. WHO. Vector control for malaria and other mosquito-borne diseases. 1995.

12. WHO. Ten years of onchocerciasis control in West Africa: review of the work of the Onchocerciasis Control Programme in the Volta River Basin area from 1974 to 1984 . Geneva; 1985. https://apps.who.int/iris/handle/10665/61819.

13. Chadee DD. Effects of "closed" houses on the Aedes aegypti eradication programme in Trinidad. Med Vet Entomol. 1988;2:193-8. doi:10.1111/j.13652915.1988.tb00072.x.

14. Curtis CF. Appropriate technology in vector control. Boca Raton: CRC Press; 1990. doi:10.1201/9781351069823.

15. Elsinga J, Van Der Veen HT, Gerstenbluth I, Burgerhof JGM, Dijkstra A, Grobusch MP, et al. Community participation in mosquito breeding site control: An interdisciplinary mixed methods study in Curaçao. Parasites Vectors. 2017;10:434. doi:10.1186/s13071-017-2371-6.

16. WHO. Communication for Behavioral Impact. Geneva; 2012. doi:10.4135/9781483346427.n79.

17. Pennington PM, Pellecer Rivera E, De Urioste-Stone SM, Aguilar T, Juárez JG. A Successful Community-Based Pilot Programme to Control Insect Vectors of Chagas Disease in Rural Guatemala. Area-Wide Integr Pest Manag. 2021;:709-27.

18. CDC. ArboNET Disease Maps. ArboNET. 2021. https://wwwn.cdc.gov/arbonet/Maps/ADB_Diseases_Map/index.html.

19. Association of State and Territorial Health Officials. Analysis of Express Legal Authorities for Mosquito Control in the United States, Washington, D.C., and Puerto Rico. 2018. https://www.epa.gov/sites/production/files/2016-. Accessed 25 Jun 2021.

20. Dickinson KL, Banacos N, Carbajal E, Dacko N, Fredregill C, Hinojosa S, et al. Public willingness to pay and social acceptability for mosquito control in Texas. Emerg Infect Dis.

21. Hargrove WL, Juárez-Carillo PM, Korc M. Healthy vinton: A health impact assessment focused on water and sanitation in a small rural town on the U.S.Mexico border. Int J Environ Res Public Health. 2015;12:3864-88. doi:10.3390/ijerph120403864. 
22. Rivera DZ. The Forgotten Americans: A Visual Exploration of Lower Rio Grande Valley Colonias. Michigan J Sustain. 2014;2. doi:10.3998/mjs.12333712.0002.010.

23. HAC. Rural Research Report: Housing in the border colonias. Washigton DC. 2013. http://www.ruralhome.org. Accessed 14 Mar 2019.

24. Tsou P-Y, Agarwal R, Tomaj A, Griffin M. Assessing health status and housing quality of families living in model subdivisions (Colonias) of the Rio Grande Valley. Pediatrics. 2018;142:514-4. doi:10.1542/PEDS.142.1_MEETINGABSTRACT.514.

25. Ward PM, Carew J. Absentee lot owners in Texas colonias: Who are they, and what do they want? Habitat Int. 2000;24:327-45. doi:10.1016/S01973975(99)00047-8.

26. Mier N, Ory MG, Zhan D, Conkling M, Sharkey JR, Burdine JN. Health-related quality of life among Mexican Americans living in colonias at the TexasMexico border. Soc Sci Med. 2008;66:1760-71. doi:10.1016/j.socscimed.2007.12.017.

27. Juarez JG, Chaves LF, Garcia-Luna SM, Martin E, Badillo-Vargas I, Medeiros MCl, et al. Variable coverage in an Autocidal Gravid Ovitrap intervention impacts efficacy of Aedes aegypti control. J Appl Ecol. 2021;:1365-2664.13951. doi:10.1111/1365-2664.13951.

28. U.S. Census Bureau. Hidalgo County, Texas. 2017. https://www.census.gov/quickfacts/hidalgocountytexas. Accessed 14 Mar 2019.

29. US Bureau of Transportation Statistics. BTS Border Crossing Annual Data. 2018. https://explore.dot.gov/t/BTS/views/BTSBorderCrossingAnnualData/BorderCrossingTableDashboard?:embed=y\&:showShareOptions=true\&:display_cour

30. Juarez JG, Garcia-Luna SM, Medeiros MCl, Dickinson KL, Borucki MK, Frank M, et al. The Eco-Bio-Social Factors That Modulate Aedes aegypti Abundance in South Texas Border Communities. Insects. 2021;12:183. doi:10.3390/insects12020183.

31. Juarez JG, Garcia-Luna S, Chaves LF, Carbajal E, Valdez E, Avila C, et al. Dispersal of female and male Aedes aegypti from discarded container habitats using a stable isotope mark-capture study design in South Texas. Sci Rep. 2020;10:6803. doi:10.1038/s41598-020-63670-9.

32. Olson MF, Ndeffo-Mbah ML, Juarez JG, Garcia-Luna S, Martin E, Borucki MK, et al. High rate of non-human feeding by Aedes aegypti reduces zika virus transmission in South Texas. Viruses. 2020;12:453. doi:10.3390/v12040453.

33. Martin E, Medeiros MCl, Carbajal E, Valdez E, Juarez JG, Gracia-Luna S, et al. Surveillance of Aedes aegypti indoors and outdoors using Autocidal Gravid Ovitraps in South Texas during local transmission of Zika virus, 2016 to 2018. Acta Trop. 2019;192 January:129-37.

doi:10.1016/j.actatropica.2019.02.006.

34. WHO. Community engagement: A health promotion guide for universal health coverage in the hands of the people. Geneva; 2020. doi:10.1007/978-3-31995717-3_300029.

35. National Academies of Sciences Engineering and Medicine. Engaging Communities, Stakeholders, and Publics. In: Genedrives on the horizon: advancing science, navigating uncertainty, and aligning research with public values. 2016. p. 121-36.

36. National Academies of Sciences Engineering and Medicine. Charting Human Values. In: Genedrives on the horizon: advancing science, navigating uncertainty, and aligning research with public values. 2016. p. 59-79.

37. WHO. WHO Community Engagement Framework for Quality, People-centred and Resilient Health Services. Geneva; 2017. https://apps.who.int/iris/bitstream/handle/10665/259280/WHO-HIS-SDS-2017.15-eng.pdf. Accessed 25 Aug 2021.

38. Grinker RR, Chambers N, Njongwe N, Lagman AE, Guthrie W, Stronach S, et al. "Communities" in Community Engagement: Lessons Learned From Autism Research in South Korea and South Africa. Autism Res. 2012;5:201-10. doi:10.1002/aur.1229.

39. Myers HF, Alvy KT, Richardson M, Arrington A, Marigna M. The effective black parenting program: A controlled research study with inner-city black families. Studio City, CA; 1990.

40. 40. Myers HF, Alvy KT, Richardson M, Arrington A, Marigna M. The effective black parenting program: A controlled research study with inner-city black families. Studio City, CA; 1990.

\section{Figures}

\section{Figure 1}

Site location of the communities involved in the Autocidal Gravid Ovitrap (AGO), Autodissemination Station (ADS) and ecological studies of Aedes aegypti in the Lower Rio Grande Valley, South Texas. A) Map of Texas highlighting Hidalgo County. B) Study communities' location within the LRGV region, AGO study= blue dots, ecological-ADS study= green dots. C) Communities involved in the AGO study. D) Communities involved in the ecological and ADS studies.

Community engagement (CE) refers to the year when recruitment of all houses within a community was conducted. The map was developed using QGIS 3.16 (https://qgis.org/en/site/) with Map data: Google, Maxar Technologies.

\section{Figure 2}

Timeline and activities carried out in the colonias of the Lower Rio Grande Valley (LRGV) in South Texas. Phase 1 shows the activities carried out during the initial approach of the project starting in September 2016. Phase 2 shows the $1^{\text {st }}$ recruitment period starting in July 2017 . Phase 3 shows the $2^{\text {nd }}$ recruitment period starting in June 2018. 
Figure 3

Information flyer used during the recruitment of the Autocidal Gravid Ovitrap project. A) Information flyer of 2017. B) Information flyer of 2018 with community suggestions incorporated. Flyers generated using PowerPoint (Microsoft, USA) and Affinity Designer 1.8 (Serif).

\section{Figure 4}

Recruitment rates for the Autocidal Gravid Ovitrap Project in colonias of the Lower Rio Grande Valley in 2017 (Green) and 2018 (Orange). Our team visited every household in each colonia up to three times to recruit them to the study. Results are presented as the percent of the households in each colonia that agreed to participate (by letting us deploy 3 AGOs on their property) after the first, second, and third visits, and in total.

\section{Figure 5}

Information flyer used during the recruitment of the ADS project for Phase 3, July 2018. Flyer generated using PowerPoint (Microsoft, USA).

\section{Figure 6}

Recruitment rates for the Ecological (Green $=2017)$ and Autodissemination Station (ADS) (Orange $=2018)$ Projects in colonias of the Lower Rio Grande Valley. Our team visited every household in each colonia up to three times to recruit them to the study. Results are presented as the percent of the households in each colonia that agreed to participate after the first, second, and third visits, and in total.

\section{Supplementary Files}

This is a list of supplementary files associated with this preprint. Click to download.

- Supplementaryinformation.docx 